Pacific Journal of Mathematics

TREE STRUCTURES IN BANACH SPACES 


\title{
ON TREE STRUCTURES IN BANACH SPACES
}

\author{
R. E. HARRELL AND L. A. KarLOVitZ
}

The purpose of this note is to relate the structure of flat Banach spaces to the "trees" of James. Based on this and some recent work of several other authors, we explore a similar relation for two further classes of spaces, namely spaces which are dual to spaces containing a subspace isomorphic to $l_{1}$ and spaces which are dual to spaces that are not separable.

A Banach space $X$ is said to be flat if the girth of its unit ball (defined by Schäffer [14] to be the infimum of the lengths of all centrally symmetric curves which lie in the surface of the unit ball) is four and if the girth is achieved by some curve (i.e., the infimum is a minimum). This is equivalent to the statement that there exists a function $g:[0,2] \rightarrow X$ such that

$$
\begin{aligned}
& \|g(t)\|=1 \text { for each } t \in[0,2], g(0)=-g(2), \\
& \text { and } g \text { is Lipschitz continuous with constant } 1 \text {. }
\end{aligned}
$$

Examples, consisting of common spaces, appear below. Some distinguished geometric properties of flat Banach spaces, including the ones which give rise to the term flat, were given by the authors in [3] and [4].

A Banach space $X$ is said to have the infinite tree property (James, [6]) if for some $\varepsilon>0, X$ contains a tree with an infinite number of branches, i.e., there are elements $x\left((2 i-1) / 2^{n}\right), i=1, \cdots 2^{n-1}$, $n=1,2, \cdots$, in the unit ball of $X$ which branch at each $x(\cdot)$ according to

$$
x\left(\frac{2 i-1}{2^{n}}\right)=\frac{1}{2} x\left(\frac{2(2 i-1)-1}{2^{n+1}}\right)+\frac{1}{2} x\left(\frac{2(2 i)-1}{2^{n+1}}\right)
$$

and

$$
\left\|x\left(\frac{2(2 i-1)-1}{2^{n+1}}\right)-x\left(\frac{2(2 i)-1}{2^{n+1}}\right)\right\| \geqq \varepsilon .
$$

The space is said to have the finite tree property (James, [6] if for some $\varepsilon>0$ and for each positive integer $N, X$ contains a tree with $N$ branchings (i.e., $n=1, \cdots, N$ ).

At present it is known that $X$ has the finite tree property if and only if $X$ is not super-reflexive (James, [8]) if and only if the girth of the unit ball of $X$ is equal to four (James and Schäffer, [9]). If 
$X$ has the infinite tree property, then $X$ contains a closed bounded convex subset which is not dentable, and hence $X$ is not reflexive; however, there exist nonreflexive spaces which do not have the infinite tree property (James, [6]).

It is our purpose to extend these uses of tree properties. As our starting point we note that if $X$ is flat, then it has the infinite tree property. (This is easy to see, and for completeness we note that it follows immediately from Theorem 1 below.) However, the converse is not true, nor is it true that a space with the infinite tree property is isomorphic to a flat Banach space. We now introduce a somewhat stronger property which will characterize flat Banach spaces.

The space $X$ is said to have the infinite supported tree property if for some $\delta>0$ there are elements $x\left((2 i-1) / 2^{n}\right), i=1, \cdots, 2^{n-1}$, $n=1,2, \cdots$, in the unit ball of $X$ and elements $h^{*}\left(k / 2^{m}\right), k=0, \cdots, 2^{m}$, $m=1,2, \cdots$, in the unit ball of the dual space $X^{*}$ so that (2) is satisfied, and

$$
\left\langle h^{*}\left(\frac{k}{2^{m}}\right), x\left(\frac{2 i-1}{2^{n}}\right)\right\rangle=\left\{\begin{array}{rll}
-\delta, & \text { if } i / 2^{n-1} \leqq k / 2^{m}, \\
\delta, & \text { if } k / 2^{m} \leqq(i-1) / 2^{n-1}
\end{array}\right.
$$

The geometric interpretation is clear. First, by (2) and (4), the $x$ 's form an infinite tree with $\varepsilon=2 \delta$. Second, for each rational $k / 2^{m} \in[0,1]$, the infinite tree is supported by two hyperplanes as follows. By (2) and (4), each point of the set $\left\{x\left((2 i-1) / 2^{n}\right)\right\}$ either lies in one of the two hyperplanes $\left\{x:\left\langle h^{*}\left(k / 2^{m}\right), x\right\rangle=\delta\right\},\left\{x:\left\langle h^{*}\left(k / 2^{m}\right), x\right\rangle=-\delta\right\}$, or it is a finite convex combination of points that do.

Theorem 1. The Banach space $X$ is isomorphic to a flat Banach space $Y$ if and only if $X$ has the infinite supported tree property.

It is known that the infinite tree property is not preserved under duality; $c_{0}$ has the property while $l_{1}$ does not (James [6]). However, the infinite supported tree property is preserved. This follows from Theorem 1 and from the fact that the dual of a flat space is again flat (Karlovitz, [10]).

CoRollary. If a Banach space $X$ has the infinite supported tree property, then its dual space $X^{*}$ also has this property. Moreover, $X^{*}$ is nonseparable.

ExAmple. The spaces $C[0,1]$ and $L^{1}[0,1]$, as well as $L^{1}[\mu]$ for $\mu$ not purely atomic, and $C_{\sigma}(K)$, for various choices of $\sigma$ and $K$, are flat (Harrell-Karlovitz, [3], [4], Schäffer, [15], and Nyikos-Schäffer, 
[13]) and therefore have the infinited supported tree property. Since $l_{1}$ fails to have the infinite tree property, both $c_{0}$ and $l_{1}$ fail to have the infinite supported tree property.

To see, in concrete terms, that $L^{1}[0,1]$ has the infinite supported tree property, choose $x\left((2 i-1) / 2^{n}\right)$ to be the normalized characteristic function of the interval $\left[(i-1) / 2^{n-1}, i / 2^{n-1}\right]$ and choose $h^{*}\left(k / 2^{m}\right)$ to be the linear functional

$$
-\int_{0}^{k / 2^{m}}(\cdot) d s+\int_{k / 2^{m}}^{1}(\cdot) d s .
$$

The following two theorems relate the infinite supported tree property to two further classes of spaces. They rely on some known facts about flat Banach spaces, on recent work of James, [7], Hagler, [2], Lindenstrauss-Zippin (see [12]), Stegall, [16], as well as on Theorem 1.

Theorem 2. Suppose $X^{*}$ is the dual of the Banach space $X$. If $X^{*}$ has the infinite supported tree property, then it is not separable, but not conversely-not even if $X$ is separable.

REMARK. It is interesting to compare Theorem 2 to the situtation involving the infinite tree property (not necessarily supported). The first part of the theorem remains true and is thus strengthened, i.e., if $X^{*}$ has the infinite tree property then it is not separable. On the other hand, the second part of the theorem is quite altered, and thus shows an important difference between supported and unsupported trees. For a recent result of Stegall [17] shows that if $X$ is separable and $X^{*}$ is not separable then $X^{*}$ has the infinite tree property.

The first assertion depends on the abovementioned fact that an infinite tree leads directly to a bounded closed convex set which is not dentable. Thus $X^{*}$ does not have the Radon-Nikodym property (Huff [5]), and hence it cannot be separable (Dunford-Pettis [1]).

THeorem 3. Suppose $X^{*}$ is the dual of the Banach space $X$. If $X$ contains a subspace isomorphic to $l_{1}$, then $X^{*}$ has the infinite supported tree property, but not conversely.

A tree property which is stronger than the infinite supported tree property and which characterizes those $X^{*}$ which are dual to a space $X$ containing a subspace isomorphic to $l_{1}$ is the subject of a forthcoming paper by the second author [11]. It is clear that the structure of other classes of spaces should be expressible in terms of trees. 
2. Proof of the theorems.

Proof of Theorem 1. Suppose first that $Y$ is a flat Banach space. Let $g:[0,2] \rightarrow Y$ be a girth curve of $Y$. Hence, if $0 \leqq s<t \leqq 2$, $2=\|g(0)-g(2)\| \leqq\|g(0)-g(s)\|+\|g(s)-g(t)\|+\|g(t)-g(2)\| \leqq$ $s+(t-s)+(2-t)=2$. Consequently,

$$
\|g(s)-g(t)\|=|s-t|, \text { for each pair } s, t \in[0,2] \text {. }
$$

For each $t \in[0,2]$, we choose a functional $f^{*}(t) \in Y^{*}$ satisfying:

$$
\left\langle f^{*}(t), g(t)\right\rangle=1 \text { and }\left\|f^{*}(t)\right\|=1 \text {. }
$$

By (5), (6) and $g(0)=-g(2)$, if $s, t \in[0,2]$,

$$
\left|\left\langle f^{*}(t), g(s)\right\rangle-1\right| \leqq\|g(s)-g(t)\|=|s-t|,
$$

and

$$
\begin{aligned}
& \left|\left\langle f^{*}(t), g(s)\right\rangle+1\right| \leqq\|g(s)+g(t)\| \\
& \quad \leqq\left\{\begin{array}{l}
\|g(s)-g(0)\|+\|g(2)-g(t)\|=s+(2-t) \\
\|g(s)-g(2)\|+\|g(0)-g(t)\|=(2-s)+t
\end{array}\right.
\end{aligned}
$$

From these inequalities we immediately derive

$$
\left\langle f^{*}(t), g(s)\right\rangle=1-|s-t| \text { for each pair } s, t \in[0,2] .
$$

We let

$$
\begin{gathered}
y\left((2 i-1) / 2^{n}\right)=2^{n-1}\left[g\left((i-1) / 2^{n-1}\right)-g\left(i / 2^{n-1}\right)\right], \\
n=1,2, \cdots, i=1, \cdots, 2^{n-1} .
\end{gathered}
$$

By (5), $\left\|y\left((2 i-1) / 2^{n}\right)\right\|=1$. It follows from (8) that

$$
\begin{aligned}
& y\left((2 i-1) / 2^{n}\right)=2^{n-1}\left[g\left(2(i-1) / 2^{n}\right)-g\left((2 i-1) / 2^{n}\right)\right] \\
& \quad+2^{n-1}\left[g\left((2 i-1) / 2^{n}\right)-g\left(2 i / 2^{n}\right)\right]=\frac{1}{2} y\left((2(2 i-1)-1) / 2^{n+1}\right) \\
& \quad+\frac{1}{2} y\left((2(2 i)-1) / 2^{n+1}\right), \text { for all } y\left((2 i-1) / 2^{n}\right) .
\end{aligned}
$$

By virtue of (7), we have

$$
\left\langle f^{*}\left(\frac{k}{2^{m}}\right), y\left(\frac{2 i-1}{2^{n}}\right)\right\rangle=\left\{\begin{aligned}
-1, & \text { if } i / 2^{n-1} \leqq k / 2^{m}, \\
1, & \text { if } k / 2^{m} \leqq(i-1) / 2^{n-1}
\end{aligned}\right.
$$

for all $y\left((2 i-1) / 2^{n}\right)$ and all $f^{*}\left(k / 2^{m}\right)$, with $m=1,2, \cdots, k=0, \cdots, 2^{m}$. Since $\left\|y\left((2 i-1) / 2^{n}\right)\right\|=\left\|f^{*}\left(k / 2^{m}\right)\right\|=1$, it follows from (9) and (10) that $Y$ has the infinite supported tree property. It is clear that the infinite supported tree property is isomorphically invariant. Therefore 
$X$ also has this property.

Suppose, conversely, that $X$ has the infinite supported tree property, i.e., suppose that $\left\{x\left((2 i-1) / 2^{n}\right): n=1,2, \cdots, i=1, \cdots, 2^{n-1}\right\}$ is a subset of the unit ball of $X$ and that $\left\{h^{*}\left(k / 2^{m}\right): m=1,2, \cdots\right.$, $\left.k=0, \cdots, 2^{m}\right\}$ is a subset of the unit ball of the dual space $X^{*}$ and suppose that (2) and (4) are satisfied. We prove that $X$ is isomorphic to a flat Banach space.

By repeated application of (2), we note that whenever $p \geqq n$,

$$
x\left((2 i-1) / 2^{n}\right)=\left(1 / 2^{p-n}\right) \sum_{j=2^{p-n_{i-2}}}^{2^{p-n_{+}}} x\left((2 j-1) / 2^{p}\right) .
$$

We define a new norm $\|\cdot\| \|$ on $X$ by

$$
\begin{aligned}
\|x\| & =\max \left\{\|x\|, \sup \left\{(1 / \delta)\left|\left\langle h^{*}\left(k / 2^{m}\right), x\right\rangle\right|: m=1,2, \cdots,\right.\right. \\
k & \left.=0, \cdots, 2^{m}\right\},
\end{aligned}
$$

where $\delta>0$ is given by (4). Clearly,

$$
\|x\| \leqq|\|x\|| \leqq(1 / \delta)\|x\|, \text { for all } x \in X \text {. }
$$

We assert that, with the new norm $\|\cdot \cdot\| \mid, X$ is flat. To prove this, we first note that by (4),

$$
\left|\left\langle h^{*}\left(i / 2^{n-1}\right), x\left((2 i-1) / 2^{n}\right)\right\rangle\right|=\delta .
$$

Moreover, by (4) and (11), $\left\langle h^{*}\left(k / 2^{m}\right), x\left((2 i-1) / 2^{n}\right)\right\rangle \leqq \delta$, for $k / 2^{m} \neq$ $i / 2^{n-1}$; thus, by $\left\|x\left((2 i-1) / 2^{n}\right)\right\| \leqq 1$ and (12), we have

$$
\left\||| x\left((2 i-1) / 2^{n}\right)\right\|=1, \quad n=1,2, \cdots, i=1, \cdots, 2^{n-1} .
$$

We now define a function $g:\left\{j / 2^{n}: n=1,2, \cdots, j=0, \cdots, 2^{n-1}\right\} \rightarrow X$ by

$$
\begin{aligned}
g\left(j / 2^{n}\right)= & -\left(1 / 2^{n+1}\right) \sum_{i=1}^{j} x\left((2 i-1) / 2^{n+2}\right) \\
& +\left(1 / 2^{n+1}\right) \sum_{i=j+1}^{2^{n+1}} x\left((2 i-1) / 2^{n+2}\right),
\end{aligned}
$$

where, of course, one of the sums may be empty. By virtue of (11), $g$ is well defined, i.e., the value $g\left(j / 2^{n}\right)$ is independent of the representation of $j / 2^{n}$. Clearly $g(0)=-g(2)$. By (13) and (14), || $g\left(j / 2^{n}\right) \| \mid \leqq 1$. Moreover, by (4) and (14), $\left\langle h^{*}\left(j / 2^{n+1}\right), g\left(j / 2^{n}\right)\right\rangle=\delta$. Hence, by (12), ||$\left|g\left(j / 2^{n}\right)\right||| \geqq(1 / \delta)\left|\left\langle h^{*}\left(j / 2^{n+1}\right), g\left(j / 2^{n}\right)\right\rangle\right|=1$. Combining the two inequalities, we have

$$
\left\||| g\left(j / 2^{n}\right)\right\|=1, \quad n=1,2, \cdots, j=0, \cdots, 2^{n+1} .
$$

Using (13) and (14) we estimate for $0 \leqq k<j \leqq 2^{n+1}$, 


$$
\begin{aligned}
& \|\| g\left(k / 2^{n}\right)-g\left(j / 2^{n}\right) \| \\
& \quad \leqq\left(2 / 2^{n+1}\right) \mid\left\|\sum_{k+1}^{j} x\left((2 i-1) / 2^{n+1}\right)\right\| \leqq(j-k) / 2^{n} .
\end{aligned}
$$

This shows that $g$ is Lipschitz continuous with constant 1 on its domain of definition $\left\{j / 2^{n}: n=1,2, \cdots, j=0, \cdots, 2^{n+1}\right\}$. Hence we can extend $g$ to the entire interval $[0,2]$ so that the extension, again denoted by $g$, is also Lipschitz continuous with constant 1 and satisfies $\|\mid g(t)\| \|=1$ for each $t \in[0,2]$. Since, moreover, $g(0)=-g(2)$, $g:[0,2] \rightarrow X$ is a girth curve for $X$ in the norm $\|\cdot \cdot\| \mid$. Hence $X$, in this norm, is flat. This finishes the proof of Theorem 1.

Proof of Corollary. It was shown in Karlovitz [10] that if $X$ is flat, then $X^{*}$ is also flat and not separable: The corollary follows from this and Theorem 1 .

Proof of Theorem 2. According to Theorem 4 of Harrell-Karlovitz [4], a flat Banach space cannot be isomorphic to any subspace of any separable dual space. By Theorem 1 it follows that $X^{*}$ is not separable.

To prove the other half of the theorem, we consider the separable space $J_{0}$ defined by James [7]. Since $J_{0}$ is also the dual of another space, it fails to have the infinite supported tree property, by virtue of the first half of the theorem. Furthermore, in LindenstraussStegall [12] it is shown that $J_{0}^{* *} \cong J_{0} \oplus l^{2}\left(2^{\aleph_{0}}\right)$. From this it follows that $J_{0}^{* *}$ fails to have the infinite supported tree property. By the Corollary it follows that $J_{0}^{*}$ also fails to have the infinite supported tree property. Finally, (James, [7]) $J_{0}^{*}$ is not separable. This finishes the proof of Theorem 2.

Proof of Theorem 3. Suppose first that $X$ contains a subspace isomorphic to $l_{1}$. Then, by Hagler [2], $X^{*}$ contains a subspace isomorphic to $L^{1}[0,1]$. As noted above, $L^{1}[0,1]$ is flat. Hence, by Theorem $1, X^{*}$ has the infinite supported tree property.

To prove the other half of the theorem, consider the linear space of all real-valued step functions $y(t), 0 \leqq t \leqq 2$. Define a norm on $Y$ by

$$
\|y\|=\sup \left[\sum_{i=1}^{n-1}\left(\int_{t_{i}}^{t_{i+1}} y\right)^{2}+\left(\int_{0}^{t_{1}} y+\int_{t_{n}}^{2} y\right)^{2}\right]^{1 / 2}
$$

where the supremum is taken over all $0 \leqq t_{1} \leqq \cdots \leqq t_{n} \leqq 2$. Now let $X$ be the completion of $Y$. It is readily seen that $X$ is isomorphic to the space defined by Lindenstrauss and Zippin, which is discussed in [12], and which is proved not to contain a subspace isomorphic 
to $l_{1}$. Moreover, $X$ is readily seen to be flat. To this end, define $h:[0,1] \rightarrow X$ by

$$
h(s)(t)=\left\{\begin{aligned}
-1 / \sqrt{2}, & 0 \leqq t \leqq s, \quad s+1 \leqq t \leqq 2 \\
1 / \sqrt{2}, & s \leqq t \leqq s+1
\end{aligned}\right.
$$

and define $g:[0,2] \rightarrow X$ by $g(s)=h(s / 2), 0 \leqq s \leqq 2$. It is readily observed that $g$ satisfies (1), and hence $X$ is flat. Therefore, by the Corollary, $X^{*}$ has the infinite supported tree property. Since $X$ does not contain a subspace isomorphic to $l_{1}$, this finishes the proof of Theorem 3.

\section{REFERENCES}

1. N. Dunford, and B. J. Pettis, Linear operators and summable functions, Trans. Amer. Math. Soc., 47 (1940), 323-392.

2. J. Hagler, Embeddings of $L^{1}$ spaces into conjugate Banach spaces, $\mathrm{Ph}$. D. Thesis (U. C. B., 1972).

2. R.E. Harrell, and L.A. Karlovitz, Nonreflexivity and the girth of spheres, Inequalities III, Academic Press, New York (1972), 121-127.

4. — The geometry of flat Banach spaces, Trans. Amer. Soc., 192 (1974), 209-218.

5. R. E. Huff, Dentability and the Radon-Nikodym property, Duke Math. J., 41 (1974), 111-114.

6. R. C. James, Some self-dual properties of normed linear spaces, Symposium on Infinite Dimensional Topology, Princeton University Press, Princeton (1972), 159-175.

7. - A conjecture about $l_{1}$ subspaces, (to appear).

8. — Super-reflexive Banach spaces, (to appear).

9. R. C. James, and J. J. Schäffer, Super-reflexivity and the girth of spheres, Israel J. Math., 11 (1972),, 398-404.

10. L. A. Karlovitz, On the duals of flat Banach spaces, Math. Ann., 202 (1973), 245-250.

11. - Tree structures and $l_{1}$ subspaces, (to appear).

12. J. Lindenstrauss, C. Stegall, Examples of spaces which do not contain $l_{1}$ and whose duals are non-separable, (to appear).

13. P. Nyikos, and J.J. Schäffer, Flat spaces of continuous functions, Studia Math., 42 (1972), 221-229.

14. J. J. Schäffer, Inner diameter, perimeter, and girth of spheres, Math. Ann., 173 (1967). 59-79.

15. - On the geometry of spheres in L-spaces, Israel J. Math., 10 (1971), 114120.

16. C. Stegall, Banach spaces whose duals contain $l_{1}(\Gamma)$ with applications to the study of dual $L_{1}(\mu)$ spaces, Trans. Amer. Math. Soc., 176 (1973), 463-477.

17. - The Radon-Nikodym property in conjugate Banach spaces, Trans. Amer. Math. Soc., (to appear).

Received July 17, 1974. First author, Departmen of Mathematics, Allegheny College, Meadville, PA 16335. Second author, Institute for Fluid Dynamics and Applied Mathematics, University of Maryland, College Park, MD 20742. Research supported in part by the National Science Foundation under Grant GP 20555. 



\section{PACIFIC JOURNAL OF MATHEMATICS}

\section{EDITORS}

RICHARD ARENS (Managing Editor) University of California

Los Angeles, California 90024

\section{R. A. Beaumont}

University of Washington Seattle, Washington 98105
J. DugundJI Department of Mathematics University of Southern Californıa Los Angeles, California 90007

D. Gilbarg aNd J. Milgram Stanford University Stanford, California 94305

\section{ASSOCIATE EDITORS}

E. F. BeCKENBACH

B. H. NeUmanN

F. Wolf

K. YoSHIDA

\section{SUPPORTING INSTITUTIONS}

UNIVERSITY OF BRITISH COLUMBIA CALIFORNIA INSTITUTE OF TECHNOLOGY UNIVERSITY OF CALIFORNIA MONTANA STATE UNIVERSITY UNIVERSITY OF NEVADA NEW MEXICO STATE UNIVERSITY OREGON STATE UNIVERSITY UNIVERSITY OF OREGON OSAKA UNIVERSITY
UNIVERSITY OF SOUTHERN CALIFORNIA STANFORD UNIVERSITY UNIVERSITY OF TOKYO UNIVERSITY OF UTAH WASHINGTON STATE UNIVERSITY UNIVERSITY OF WASHINGTON AMERICAN MATHEMATICAL SOCIETY NAVAL WEAPONS CENTER 


\section{Pacific Journal of Mathematics \\ Vol. 59, No. 1 \\ May, 1975}

Shashi Prabha Arya and M. K. Singal, More sum theorems for topological

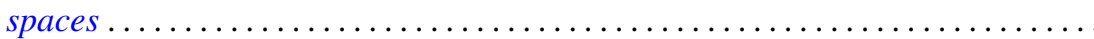

Goro Azumaya, F. Mbuntum and Kalathoor Varadarajan, On M-projective and

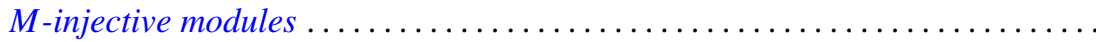

Kong Ming Chong, Spectral inequalities involving the infima and suprema of

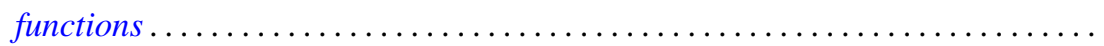

Alan Hetherington Durfee, The characteristic polynomial of the monodromy .......

Emilio Gagliardo and Clifford Alfons Kottman, Fixed points for orientation

preserving homeomorphisms of the plane which interchange two points ......

Raymond F. Gittings, Finite-to-one open maps of generalized metric spaces .......

Andrew M. W. Glass, W. Charles (Wilbur) Holland Jr. and Stephen H. McCleary,

$a^{*}$-closures of completely distributive lattice-ordered groups .............

Matthew Gould, Endomorphism and automorphism structure of direct squares of

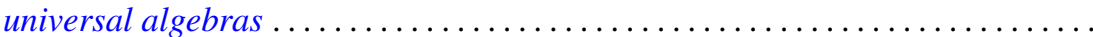

R. E. Harrell and Les Andrew Karlovitz, On tree structures in Banach spaces .....

Julien O. Hennefeld, Finding a maximal subalgebra on which the two Arens

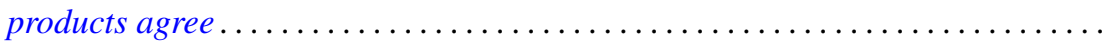

William Francis Keigher, Adjunctions and comonads in differential algebra .......

Robert Bernard Kelman, A Dirichlet-Jordan theorem for dual trigonometric

series

Allan Morton Krall, Stieltjes differential-boundary operators. III. Multivalued operators-linear relations...

Hui-Hsiung Kuo, On Gross differentiation on Banach spaces .

Tom Louton, A theorem on simultaneous observability ...

Kenneth Mandelberg, Amitsur cohomology for certain extensions of rings of algebraic integers.

Coy Lewis May, Automorphisms of compact Klein surfaces with boundary . . .

Peter A. McCoy, Generalized axisymmetric elliptic functions .

211

Muril Lynn Robertson, Concerning Siu's method for solving $y^{\prime}(t)=F(t$, $y(g(t)))$. .

Richard Lewis Roth, On restricting irreducible characters to normal

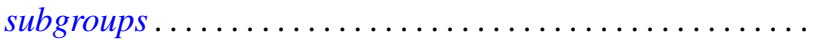

Albert Oscar Shar, $P$-primary decomposition of maps into an $H$-space .

Kenneth Barry Stolarsky, The sum of the distances to certain pointsets on the unit circle.

Bert Alan Taylor, Components of zero sets of analytic functions in $C^{2}$ in the unit ball or polydisc

Michel Valadier, Convex integrands on Souslin locally convex spaces ...

Januario Varela, Fields of automorphisms and derivations of $C$

Arnold Lewis Villone, A class of symmetric differential operators with deficiency

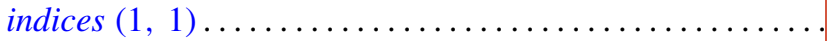

